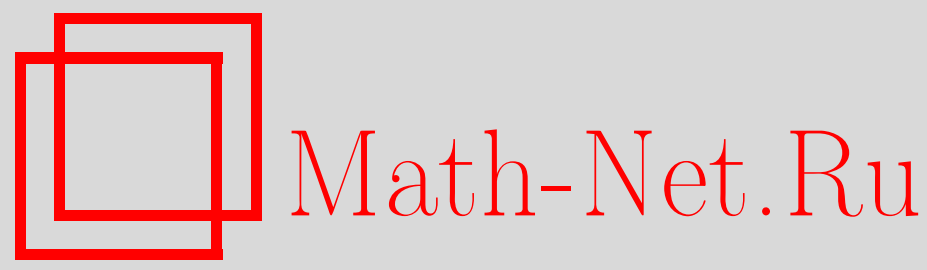

В. П. Маслов, Аксиомы нелинейного осреднения в финансовой математике и динамика курса акций, Теория вероятн. и ее примен., 2003, том 48, выпуск 4, 800-810 DOI: https://doi.org/10.4213/tvp258

Использование Общероссийского математического портала Math-Net.Ru подразумевает, что вы прочитали и согласны с пользовательским соглашением

http://www . mathnet.ru/rus/agreement

Параметры загрузки:

IP : 52.87 .193 .239

26 апреля 2023 г., 12:28:53

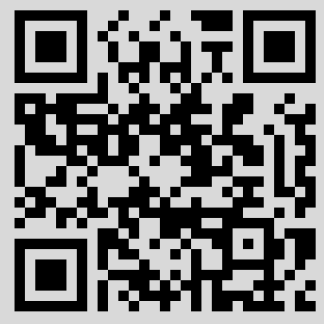


7. Sato K.-I. Lévy Processes and Infinitely Divisible Distributions. Cambridge: Cambridge Univ. Press, 1999, $486 \mathrm{p}$.

8. Якьмив $A$. Л. Явные неравенства для асимптотики субэкспоненциальных безгранично делимых распределений. - Матем. заметки, 2000, т. 67, № 2, с. 295-301.

9. Baltrūnas A., Omey $E$. The rate of convergence for subexponential distributions. Liet. Mat. Rink., 1998, v. 38, № 1, p. 1-18.

10. Omey E., Willekens E. Second-order behaviour of distributions subordinate to a distribution with finite mean. - Comm. Statist. Stochastic Models, 1987, v. 3, № 3, p. 311-342.

11. Willekens E. The structure of the class of subexponential distributions. - Probab. Theory Related Fields, 1988, v. 77, № 4, p. 567-581.

Поступила в редакцию

30.I.2002

(C) 2003 r.

МАСЛОВ В. П.*

\title{
АКСИОМЫ НЕЛИНЕЙНОГО ОСРЕДНЕНИЯ В ФИНАНСОВОЙ МАТЕМАТИКЕ И ДИНАМИКА КУРСА АКЦИЙ ${ }^{1)}$
}

\author{
К 80-летию Б. А. Севастьянова
}

\begin{abstract}
При наличии фактора неопределенности, заключающегося в том, что некоторая величина $X$ принимает не одно значение, а множество значений $x_{1}, \ldots, x_{n}$, по ним обычно проводится осреднение с некоторыми коэффициентами $\alpha_{i}$ (мерами), такими, что $\sum_{i=1}^{n} \alpha_{i}=1: y=\sum \alpha_{i} x_{i}$. Когда речь идет о рынке ценных бумаг, возникает нелинейное осреднение величины $y$. В работе рассматривается осреднение вида $f(y)=\sum \alpha_{i} f_{i}\left(x_{i}\right)$. Исходя из четырех естественных аксиом доказывается, что либо выполняется вышеупомянутое линейное осрецнение, либо $y=\ln \sum_{i=1}^{n} e^{x_{i}}$. Приводится пример пробоя курса акций при таком суммировании.
\end{abstract}

Ключевые слова и фразы: математическое ожидание, фактор неопределенности, значение случайной величины, доход, банки, акции, финансовая динамика, пробой курса акций.

Для того чтобы уловить усредненные колебания курса акций, который может меняться очень быстро, например, за одну ночь или даже в течение одного часа, приходится осреднять их цены и определять индексы, которые меняются достаточно плавно. Например, индексы одной отрасли, к тому же осредненные по достаточно большому интервалу времени, могут меняться достаточно плавно в течение на порядок большего времени.

Естественно осреднять доходы, просто взяв арифметическое среднее. Более гибкое осреднение применяется, когда берутся взвешенные суммы. Пусть $x_{i}$, $i=1, \ldots, n,-$ доходы, $\alpha_{i}$ - «веса», $\sum_{i=1}^{n} \alpha_{i}=1$, тогда

$$
y=\sum \alpha_{i} x_{i}
$$

осредненный доход (см., например, [1, с. 109], [2, с. 28], [3, с. 119], [4, с. 31]).

Однако всем известно, что суммирование доходов нелинейно. Например, если сумма купленных акций предприятия превышает $50 \%$ всех акций, то владелец этих

* Московский государственный университет им. М. В. Ломоносова, физический факультет, кафедра квантовой статистики, Ленинские горы, 119992 Москва, Россия; e-mail: victor@maslov.msk.su 
акций становится по существу хозяином предприятия, а это может значить, что его суммарный доход изменяется скачком.

Возникает вопрос: как определить капитал данного физического лица? За рубежом его естественно сопоставить не с теми деньгами, которые у него лежат в сейфе, а с тем кредитом, который он может получить. Там все в основном живут в долг, даже государства. A этот кредит не является линейной функцией дохода.

Он основывается на кредитоспособности заемщика, при определении которой учитываются, в частности, следуюшие показатели: 1) постоянный доход (официальная зарплата, рента, пенсия и т.п.); 2) статус занятости (при этом наиболее выгоден полный рабочий день); 3) возраст (наиболее предпочитаемый возраст от 22 до 50); 4) количество материально зависимых лиц (несовершеннолетние дети, инвалиды и т.п.); 5) гражданство; 6) другие займы, кредитные карты, закладные и неоплаченные задолженности; 7) кредитная история. Она составляется из предыдущих кредитов и кредитных карт претендента. Заемщик, который ранее имел несколько кредитных карт и займов и регулярно вовремя погашал их, скорее всего будет иметь более высокую оценку кредитоспособности, чем претендент, никогда не имевший кредитных карт и не бравший ссуд, или претендент, не погасивший задолженность вовремя.

Небольшие частые увеличения дохода могут привести к существенному увеличению кредита.

Нелинейными, как известно, являются и «функция полезности», и «функция предпочтения» [5]-[7]. Поэтому, вообще говоря, линейную формулу суммы (1) нужно заменить некоторой нелинейной, т.е. сделать нелинейные замены как самой функции, так и переменных $x_{i}$. Итак рассмотрим следуюшее неявное уравнение относительно «нелинейной суммы» $y^{\prime}$ от доходов $x_{i}^{\prime}$ :

$$
f\left(y^{\prime}\right)=\sum_{i} \alpha_{i} f_{i}\left(x_{i}^{\prime}\right) .
$$

Прежде всего естественно считать, что если сумма состоит из одного элемента $x_{i_{0}}^{\prime}$, то $y^{\prime}=x_{i_{0}}^{\prime}$. Иначе говоря, если $\alpha_{i}=0$ при $i \neq i_{0}$ и $\alpha_{i}=1$ при $i=i_{0}$, то $y^{\prime}=x_{i_{0}}^{\prime}$. (Назовем это аксиомой 1 - основной аксиомой.) Это существенно сужает рассматриваемый класс нелинейных преобразований. Мы получаем, что

$$
f\left(y^{\prime}\right)=\sum_{i} \alpha_{i} f\left(x_{i}^{\prime}\right)
$$

Или, в предположении, что функция $f$ является выпуклой и, значит, имеет обратную $f^{-1}(f)=1$,

$$
y^{\prime}=f^{-1}\left(\sum_{i} \alpha_{i} f\left(x_{i}^{\prime}\right)\right) .
$$

Такое суммирование уже является некоторой новой «арифметикой» [17].

Итак пусть $f(x) \in C(\mathbf{R})$ - некоторая функция, имеющая обратную функцию $f^{-1}(x)$. Каждой функции $f$ сопоставим пару бинарных операций $\oplus_{f}$ и $\odot_{f}$ на множестве действительных чисел $\mathbf{R}$, которые определим следующим образом:

$$
\begin{aligned}
& a \oplus_{f} b=f^{-1}(f(a)+f(b)), \\
& a \odot_{f} b=f^{-1}(f(a) f(b)) .
\end{aligned}
$$

Покажем, что выполнены следующие условия:

1) операции $\oplus_{f}$ и $\odot_{f}$ являются ассоциативными, т.е. для любой тройки чисел $a, b, c \in \mathbf{R}$ выполняются равенства

$$
\begin{aligned}
& \left(a \oplus_{f} b\right) \oplus_{f} c=a \oplus_{f}\left(b \oplus_{f} c\right), \\
& \left(a \odot_{f} b\right) \odot_{f} c=a \odot_{f}\left(b \odot_{f} c\right) ;
\end{aligned}
$$

2) операция $\oplus_{f}$ является коммутативной, т.е. для любой пары чисел $a, b \in \mathbf{R}$ выполняется равенство

$$
a \oplus_{f} b=b \oplus_{f} a
$$


3) операция $\odot_{f}$ является дистрибутивной по отношению к операции $\oplus_{f}$, т.е. для любой тройки чисел $a, b, c \in \mathbf{R}$ выполняются равенства

$$
\begin{aligned}
& \left(a \odot_{f} b\right) \oplus_{f}\left(a \odot_{f} c\right)=a \odot_{f}\left(b \oplus_{f} c\right), \\
& \left(b \odot_{f} a\right) \oplus_{f}\left(c \odot_{f} a\right)=\left(b \oplus_{f} c\right) \odot_{f} a .
\end{aligned}
$$

Для доказательства этого утверждения достаточно непосредственно проверить выполнение равенств (7)-(11). Мы ограничимся проверкой выполнения равенства (10). Согласно (5) и (6) левая часть этого равенства имеет вид

$$
\begin{aligned}
\left(a \odot_{f} b\right) \oplus_{f}\left(a \odot_{f} c\right) & =f^{-1}\left(f\left(a \odot_{f} b\right)+f\left(a \odot_{f} c\right)\right) \\
& =f^{-1}(f(a) f(b)+f(a) f(c)) .
\end{aligned}
$$

Правая часть равенства (10) согласно определению операций $\oplus_{f}$ и $\odot_{f}$ примет вид

$$
a \odot_{f}\left(b \oplus_{f} c\right)=f^{-1}\left(f(a) f\left(b \oplus_{f} c\right)\right)=f^{-1}(f(a)(f(b)+f(c))) .
$$

Так как выражения (12) и (13) совпадают, то таким образом выполнение равенства (10) для любой тройки чисел $a, b, c \in \mathbf{R}$ доказано. Справедливость равенств (7)-(9) и (11) доказывается аналогично. Заметим еще, что операция $\odot_{f}$ также является коммутативной, т.е. для нее выполняется равенство, аналогичное (9).

Та арифметика (полукольцо), которая у нас получилась, является как бы отображением реальной обычной арифметики, которое осуществляется в коллективном психологическом восприятии общества. Это напоминает теорию Маха, который предлагал изучать не реальный мир, а его отображение в нашем сознании. Психология общества, снабженного современными средствами информации, может многократно эмоционально увеличить выигрыш или проигрыш данного конкретного лица. $\mathrm{He}$ очень значительное увеличение дохода может поставить данное лицо на некоторую новую ступень в иерархии общества. Мы уходим таким образом от реальной объективной арифметики (и теории вероятностей) в некий субъективный мир, сформированный в нашем общественном сознании (психологии общества), но не хаотический, а также достаточно стройный. Это и есть основное допущение данной работы, так как остальные аксиомы, определяющие $\alpha_{i}$ и вид функции $f$, достаточно естественны и очевидны. Их применение дает альтернативу всего из двух «арифметик», двух функций $f$ - «объективной» линейной функции, т.е. осреднения дохода как арифметического среднего (что очевидно каждому школьнику), и единственной нелинейной функции, которая удовлетворяет приводимым ниже аксиомам.

В простейшем случае доход равен частотному математическому ожиданию (см. [10]-[17]), умноженному на число испытаний $N$, т.е.

$$
x_{i}=\sum_{j=1}^{n} \lambda_{j} p_{j}^{(i)} N,
$$

где $\lambda_{1}, \ldots, \lambda_{n}-$ значения случайной величины, $p_{j}$ - частотные вероятности $N_{j} / N$, $N_{j}$ - число исходов, отвечающих $\lambda_{j}$. Величина $\lambda_{j}$ может играть роль цены (например, цены выигрыша), процента $j$-го банка и т.д.

Весовые коэффициенты $\alpha_{i}$ в выбранной заранее модели не зависят от значений $\lambda_{j}$ и от того подмножества множества $\left\{x_{i}\right\}$, которое мы рассматриваем. Иначе сравнение разных осреднений невозможно (см. [1]-[4]) (точно так же как можно сравнивать только средние значения двух случайных величин, взятые по одной мере). Мы воспользуемся этим обстоятельством (аксиома 2), чтобы определить их для нашей «модели». У нас $x_{i}$ - это деньги, и мы вычислим эти коэффициенты исходя из известной пословицы: «деньги не пахнут» (аксиома 3). А именно: если имеются разные купюры одного достоинства и мы их поменяли местами в пачке купюр, то результат от этого не изменится.

Пример. Пусть вкладчик имеет две купюры, пронумерованные числами 1 и 2 , кроме того пусть число банков, в которые он может вложить эти купюры, равно двум. В этом случае физически различные варианты вклада следующие: 1) обе купюры вложены в первый банк; 2) обе купюры вложены во второй банк; 3) купюра с номером 1 вложена в первый банк, а купюра с номером 2 - во второй; 4) купюра с 
номером 1 вложена во второй банк, а купюра с номером 2 - в первый. С финансовой точки зрения в данном случае только три различных варианта вклада, поскольку третий и четвертый варианты вклада являются неразличимыми и представляют собой один вариант, так как они отличаются только тем, что купюры одного достоинства поменяли местами, а это никак не отразится на доходах вкладчика и банков.

Теперь рассмотрим некоторое подмножество множества $\left\{x_{i}\right\}, i=1, \ldots, n$. Иначе говоря, $\alpha_{i}$, не принадлежацие этому подмножеству, полагаем равными нулю. Это подмножество, которое мы осредняем с помошью взвешенных сумм, часто называют «природной неопределенностью» [8]. В математической статистике это означает, например, что вероятности не определены, а лежат в некотором интервале. Тогда метод «взвешенных сумм» является некоторым способом осреднения и оценки этих неопределенностей.

Перейдем к подсчету коэффициентов $\alpha_{i}$ для нашей «модели». На самом деле купюры подчиняются статистике Бозе-Эйнштейна, и подсчет коэффициентов справедлив для любых объектов, подчиняющихся этой статистике. Для этого положим

$$
\lambda_{1}=\lambda_{2}=\cdots=\lambda_{G_{1}}, \quad \lambda_{G_{1}+1}=\lambda_{G_{1}+2}=\cdots=\lambda_{n} \neq \lambda_{G_{1}}
$$

и подсчитаем коэффициенты в этом частном примере. Доход (14) принимает вид

$$
x_{i}=\lambda_{1} \sum_{j=1}^{G_{1}} N_{j}^{(i)}+\lambda_{2} \sum_{j=G_{1}+1}^{n} N_{j}^{(i)} .
$$

Выражение (4) записывается следующим образом:

$$
y=f^{-1}\left(\sum_{\{N\}} \alpha(\{N\})\left(\lambda_{1} \tilde{N}_{1}(\{N\})+\lambda_{2} \widetilde{N}_{2}(\{N\})\right)\right),
$$

где $\{N\}$ - набор целых неотрицательных чисел $N_{1}, \ldots, N_{n}$, удовлетворяющих условию

$$
\sum_{j=1}^{n} N_{j}=N
$$

а $\tilde{N}_{1}(\{N\})$ и $\tilde{N}_{2}(\{N\})$ выражаются через $\{N\}$ так:

$$
\tilde{N}_{1}(\{N\})=\sum_{j=1}^{G_{1}} N_{j}, \quad \tilde{N}_{2}(\{N\})=\sum_{j=G_{1}+1}^{n} N_{j} .
$$

Из аксиомы 2 следует, что веса $\alpha(\{N\})$ не зависят от $\lambda_{1}$ и $\lambda_{2}$. Положим, что $\lambda_{1}=\lambda_{2}$, в таком случае все значения дохода (16) совпадают, а из аксиомы 3 следует, что соответствующие им веса равны. Поэтому веса не зависят от $\{N\}$ :

$$
\alpha(\{N\})=A,
$$

где

$$
A=\left(\sum_{\{N\}} 1\right)^{-1}
$$

Сумма в (17) принимает вид

$$
\sum_{\{N\}} \alpha(\{N\})\left(\lambda_{1} \tilde{N}_{1}(\{N\})+\lambda_{2} \tilde{N}_{2}(\{N\})\right)=A \sum_{\widetilde{N}_{1}=0}^{N} C\left(\tilde{N}_{1}, G_{1}\right) C\left(\tilde{N}_{2}, G_{2}\right)\left(\lambda_{1} \tilde{N}_{1}+\lambda_{2} \tilde{N}_{2}\right),
$$

где в правой части равенства $\tilde{N}_{2}=N-\tilde{N}_{1}, G_{2}=n-G_{1}$, а $C(\tilde{N}, G)$ выражаются следующим образом:

$$
C(N, G)=\sum_{N_{1}+\cdots+N_{G}=\tilde{N}} 1 .
$$


Имеет место тождество

$$
C(N, G)=\frac{(N+G-1) !}{(G-1) ! N !}
$$

Для доказательства этого тождества рассмотрим функцию $1 /(1-z)^{G}$. При $|z|<1$ эта функция представляется в виде абсолютно сходяшегося ряда

$$
\frac{1}{(1-z)^{G}}=\sum_{K=0}^{\infty} \frac{(K+G-1) !}{(G-1) ! K !} z^{K}
$$

Воспользуемся следуюшими равенствами:

$$
\begin{aligned}
\frac{1}{(1-z)^{G}} & =\left(\frac{1}{(1-z)}\right)^{G}=\left(\sum_{K=0}^{\infty} z^{K}\right)^{G} \\
& =\sum_{K_{1}=0}^{\infty} \cdots \sum_{K_{G}=0}^{\infty} z^{K_{1}+\cdots+K_{G}}=\sum_{K=0}^{\infty} C(K, G) z^{K} .
\end{aligned}
$$

Поскольку разложение функции $1 /(1-z)^{G}$ в степенной ряд единственно, то из (25) и (26) следует (24). Учтем (21) и окончательно получим из (22) следующее выражение:

$$
y=\frac{(n-1) ! N !}{(n+N-1) !} \sum_{\widetilde{N}_{1}=0}^{N} \frac{\left(G_{1}+\widetilde{N}_{1}-1\right) !}{\left(G_{1}-1\right) ! \widetilde{N}_{1} !} \frac{\left(G_{2}+\widetilde{N}_{2}-1\right) !}{\left(G_{2}-1\right) ! \widetilde{N}_{2} !}\left(\lambda_{1} \widetilde{N}_{1}+\lambda_{2} \widetilde{N}_{2}\right) .
$$

Коэффициенты (24) совпадают с кратностью вырождения системы из $N$ бозонов, которые находятся на $G$-кратно вырожденном уровне.

Доказательство того, что веса имеют вид (20), в общем случае проводится аналогично. Таким образом, для денежных единиц, так же как и вообще для объектов, подчиняюшихся Бозе-статистике, для случая, когда $\lambda_{k} \neq \lambda_{j}$ при $k \neq j$, все коэффициенты $\alpha_{i}$ с точностью до нормировки равны единице. В случае, когда функция $f$ линейна, мы приходим к очевидному результату: в качестве среднего дохода надо брать среднее арифметическое. Иначе говоря, доходы нужно просто складывать.

Вопрос, какую функцию $f$ выбрать и из каких соображений выбирать «арифметику», зависит от глобальной ситуации в стране и от взаимоотношений между различными стратами обшества (см. [9]). Однако для устоявшейся системы довольно очевидно, что должна быть выполнена следующая аксиома.

Если к $\lambda_{k}$ прибавить одну и ту же величину $\omega$, то каждый доход $x_{i}$ вида (14) увеличится на одну и ту же величину $N \omega$.

Очевидно, что нелинейное осреднение доходов $x_{i}$ в нормальных условиях также должно увеличиться на эту величину. Мы примем этот факт как аксиому 4.

Покажем, что эта аксиома приводит к однозначному решению в нелинейном случае, т.е. ей удовлетворяет естественно линейный случай (арифметическое среднее) и единственная с точностью до одной и той же константы, на которую мы можем умножить все доходы $x_{i}$, нелинейная функция.

На самом деле доходы $x_{i}$ исчисляются в какой-либо валюте и, вообще говоря, должны умножаться на некоторую величину $\beta$, которая отвечает покупательной способности этой валюты, так что указанная константа (параметр $\beta$ ) изначально должна входить в определение дохода. А следовательно мы утверждаем, что существует единственная нелинейная функция, которая удовлетворяет аксиоме 4. Докажем это.

Если функция $f(x)$ имеет вид

$$
f(x)=C \exp (D x)+B
$$

где $C, D \neq 0, B$ - числа, не зависящие от $x$, то легко проверить, что аксиома 4 выполняется. Докажем, что никакие функции $f(x)$ другого вида не удовлетворяют этой аксиоме.

Воспользуемся тем, что $f(x)$ не зависит от $n, N$, и рассмотрим случай $n=2$, $N=1$. В этом случае из аксиомы 4 и формулы (17) следует, что для любых чисел $\lambda_{1}, \lambda_{2}, \omega$ выполняется равенство

$$
f^{-1}\left(\frac{1}{2}\left(f\left(\lambda_{1}+\omega\right)+f\left(\lambda_{2}+\omega\right)\right)\right)=\omega+f^{-1}\left(\frac{1}{2}\left(f\left(\lambda_{1}\right)+f\left(\lambda_{2}\right)\right)\right) .
$$


Продифференцируем левую и правую части равенства (29) по $\omega$ и положим $\omega=0$. Получим, что для любых $\lambda_{1}$ и $\lambda_{2}$ выполняется равенство

$$
\frac{1}{2}\left(f^{\prime}\left(\lambda_{1}\right)+f^{\prime}\left(\lambda_{2}\right)\right)=f^{\prime}\left(f^{-1}\left(\frac{1}{2}\left(f\left(\lambda_{1}\right)+f\left(\lambda_{2}\right)\right)\right)\right) .
$$

Теперь продифференцируем правую и левую части равенства (30) по $\lambda_{1}$. Получим равенство, которое приводится к следуюшему виду:

$$
\frac{f^{\prime \prime}\left(\lambda_{1}\right)}{f^{\prime}\left(\lambda_{1}\right)}=\frac{f^{\prime \prime}\left(f^{-1}\left(\frac{1}{2}\left(f\left(\lambda_{1}\right)+f\left(\lambda_{2}\right)\right)\right)\right)}{f^{\prime}\left(f^{-1}\left(\frac{1}{2}\left(f\left(\lambda_{1}\right)+f\left(\lambda_{2}\right)\right)\right)\right)} .
$$

Дифференцируя левую и правую части равенства (30) по $\lambda_{2}$, получим равенство (31), у которого в левой части $\lambda_{1}$ заменено на $\lambda_{2}$. Таким образом, окончательно получим равенство

$$
\frac{f^{\prime \prime}(1)}{f^{\prime}\left(\lambda_{1}\right)}=\frac{f^{\prime \prime}\left(\lambda_{2}\right)}{f^{\prime}\left(\lambda_{2}\right)}
$$

Поскольку равенство (32) должно выполняться для любых значений $\lambda_{1}$ и $\lambda_{2}$ и эти переменные являются независимыми, то, дифференцируя его правую и левую части по $\lambda_{1}$, получим уравнение

$$
\frac{d}{d \lambda_{1}} \frac{f^{\prime \prime}\left(\lambda_{1}\right)}{f^{\prime}\left(\lambda_{1}\right)}=0
$$

Из этого уравнения следует, что

$$
\frac{f^{\prime \prime}\left(\lambda_{1}\right)}{f^{\prime}\left(\lambda_{1}\right)}=\beta,
$$

где $\beta$ - некоторое число, не зависящее от $\lambda_{1}$. Решения дифференциального уравнения (34) при $\beta=0$ имеют вид

$$
\varphi(x)=C x+B
$$

а при $\beta \neq 0$ решения уравнения (34) имеют вид

$$
\varphi(x)=C \exp (\beta x)+B
$$

где в (35) и (36) $C \neq 0$ и $B-$ некоторые постоянные. Легко убедиться, что подстановка функции (36) в (4) приводит к выражению, которое не зависит от $C$ и $B$, поэтому их значения несущественны и будем дальше считать, что $C=1, B=0$. Решений, отличных от (35) и (36), уравнение (34) не имеет, поэтому единственность с точностью до параметра $\beta$ функции $f(x)$ в нелинейном случае доказана.

Пример. Пусть выполнены условия (15). Рассмотрим случай, когда множество $\left\{x_{i}\right\}$ состоит из двух значений: $x_{0}=\lambda_{1} \widetilde{N}_{1}^{0}+\lambda_{2} \widetilde{N}_{2}^{0}$ и $x_{1}=\lambda_{1} \widetilde{N}_{1}^{1}+\lambda_{2} \widetilde{N}_{2}^{1}$. Тогда тем же способом, которым была получена формула (27), и учитывая следствие аксиомы 4, получим, что финансовое осреднение имеет вид:

$$
\begin{aligned}
y=\frac{1}{\beta} \ln ( & A\left(\frac{\left(\widetilde{N}_{1}^{0}+G_{1}-1\right) !}{\widetilde{N}_{1}^{0} !\left(G_{1}-1\right) !} \frac{\left(\widetilde{N}_{2}^{0}+G_{2}-1\right) !}{\widetilde{N}_{2}^{0 !}\left(G_{2}-1\right) !} \exp \left(\beta\left(\lambda_{1} \widetilde{N}_{1}^{0}+\lambda_{2} \widetilde{N}_{2}^{0}\right)\right)\right. \\
& \left.\left.+\frac{\left(\widetilde{N}_{1}^{1}+G_{1}-1\right) !}{\widetilde{N}_{1}^{1} !\left(G_{1}-1\right) !} \frac{\left(\widetilde{N}_{2}^{1}+G_{2}-1\right) !}{\widetilde{N}_{2}^{1} !\left(G_{2}-1\right) !} \exp \left(\beta\left(\lambda_{1} \widetilde{N}_{1}^{1}+\lambda_{2} \widetilde{N}_{2}^{1}\right)\right)\right)\right)
\end{aligned}
$$

где

$$
A^{-1}=\frac{\left(\widetilde{N}_{1}^{0}+G_{1}-1\right) !}{\widetilde{N}_{1}^{0} !\left(G_{1}-1\right) !} \frac{\left(\widetilde{N}_{2}^{0}+G_{2}-1\right) !}{\widetilde{N}_{2}^{0} !\left(G_{2}-1\right) !}+\frac{\left(\widetilde{N}_{1}^{1}+G_{1}-1\right) !}{\widetilde{N}_{1}^{1} !\left(G_{1}-1\right) !} \frac{\left(\widetilde{N}_{2}^{1}+G_{2}-1\right) !}{\widetilde{N}_{2}^{1} !\left(G_{2}-1\right) !} .
$$

Величина, стоящая в каждой экспоненте, в физике называется свободной энергией бозе-газа, деленной на температуру, если $\beta=1 / T$, где $T$ - температура. 
В случае, когда множество $x_{i}$ содержит все значения (16), формула для финансового осреднения имеет вид:

$$
\begin{aligned}
\mathbf{E}=\frac{1}{\beta} \ln \left(\frac{(n-1) ! N !}{(n+N-1) !} \sum_{\widetilde{N}_{1}=0}^{N} \frac{\left(G_{1}+\widetilde{N}_{1}-1\right) !}{\left(G_{1}-1\right) ! \widetilde{N}_{1} !} \frac{\left(G_{2}+\widetilde{N}_{2}-1\right) !}{\left(G_{2}-1\right) ! \widetilde{N}_{2} !}\right. \\
\left.\quad \times \exp \left(\beta\left(\lambda_{1} \tilde{N}_{1}+\lambda_{2} \widetilde{N}_{2}\right)\right)\right) .
\end{aligned}
$$

Далее будем называть это финансовое осреднение глобальныц финансовыц осреднением.

Запишем финансовое осреднение (39) в следующем виде:

$$
\mathbf{E}=\ln \left(\sum_{\{\tilde{N}\}} \exp (F(\{\tilde{N}\}))\right)
$$

где $F(\{\tilde{N}\})$ имеет вид

$$
F(\{\tilde{N}\})=\beta \sum_{i=1}^{k} \omega_{i} \widetilde{N}_{i}+\sum_{i=1}^{k} \ln \left(\frac{\left(G_{i}+\widetilde{N}_{i}-1\right) !}{\left(G_{i}-1\right) ! \widetilde{N}_{i} !}\right)-\ln \left(\frac{(n+N-1) !}{(n-1) ! N !}\right) .
$$

Мы предположим, что рынок стабилен и $\left\{\lambda_{i}\right\}$ не меняются в некотором промежутке времени.

Мы будем изучать поведение недальновидных игроков, которые хотят в данный момент совершить выгодную сделку и не думают о глобальном выигрыше в будущем, что может быть связано с намеренным отказом от сиюминутной выгоды (это скорее инвесторы, чем трейдеры). Эти моменты мы выбираем достаточно близкими, имея в виду, что рынок остался стабильным и $\left\{\lambda_{i}\right\}$ не изменились во все моменты времени, когда шаг за шагом играюший старается увеличить свой (осредненный финансовый) доход. Он, не делая расчет на будушее, на каждом шаге хочет получить доход, продавая или покупая не более двух акций.

Эта задача отличается от той, которую обычно ставят - выбрать наилучшую стратегию в целом. Большинство держателей акций или вкладчиков в банки придерживаются простой тактики - не много нажиться, продав пару акций или переложив деньги в другой банк. Эти деньги ему нужны «сегодня» на данном шаге, и он не рискует ради того, чтобы получить больше через несколько шагов, а «сегодня» немного проиграть. Именно за счет таких «наивных» вкладчиков обогащаются те, кто играет на повышение или понижение и выбирает хитрую далеко идущую стратегию. Нас интересуют те метаморфозы, которые происходят за счет таких наивных игроков, поскольку их большинство на рынке ценных бумаг.

Предположим, что в начальный момент наивный игрок сделал $\left\{\tilde{N}^{a}\right\}$ вкладов в $a$ банков (или купил $\left\{\tilde{N}^{a}\right\}$ акций).

Далее он делает шаги, продавая или покупая по паре акций, так, чтобы его осредненный финансовый доход увеличился. Постепенно он придет к некоторому новому набору $\left\{\widetilde{N}^{b}\right\}$ акций.

Пусть $\left\{\widetilde{N}^{a}\right\}$ и $\left\{\widetilde{N}^{b}\right\}-$ некоторые наборы исходов (вкладов). Будем говорить, что от набора $\left\{\widetilde{N}^{a}\right\}$ можно подняться до набора $\left\{\widetilde{N}^{b}\right\}$, если существует такая последовательность наборов исходов (вкладов) $\left\{\tilde{N}^{\alpha}\right\}$, где $\alpha=1, \ldots, l$, для которой выполняются следуюшие условия:

$$
\begin{aligned}
\left\{\tilde{N}^{1}\right\}=\left\{\tilde{N}^{a}\right\}, & \left\{\tilde{N}^{l}\right\}=\left\{\tilde{N}^{b}\right\}, \\
F\left(\left\{\tilde{N}^{\alpha}\right\}\right) \leqslant F\left(\left\{\tilde{N}^{\alpha+1}\right\}\right) & \text { при всех } \quad \alpha=1, \ldots, l-1, \\
\sum_{i=1}^{k}\left|\tilde{N}_{i}^{\alpha+1}-\tilde{N}_{i}^{\alpha}\right|=2 & \text { при всех } \quad \alpha=1, \ldots, l-1 .
\end{aligned}
$$


Обозначим через $I\left(\left\{\widetilde{N}^{0}\right\}\right)$ множество всех наборов вкладов $\{\tilde{N}\}$, до которых можно подняться от набора вкладов $\left\{\widetilde{N}^{0}\right\}$.

Локальным максимальным финансовым осреднением, отвечающим набору вкладов $\left\{\tilde{N}^{0}\right\}$, будем называть следуюшее выражение:

$$
\mathrm{E}_{\left\{\widetilde{N}^{0}\right\}}=\frac{1}{\beta} \ln \left(\sum_{\{\widetilde{N}\} \in I\left(\left\{\tilde{N}^{0}\right\}\right)} \exp (F(\{\tilde{N}\}))\right) .
$$

Рассмотрим пример, в котором будет установлено явление «пробоя курса акций» для локальных максимальных финансовых осреднений в пределе при $N \rightarrow \infty$.

Рассмотрим следующую финансовую модель. Вкладчик имеет $N$ одинаковых купюр, которые может вложить в две группы банков, первая группа состоит из $G_{1}$ банков, каждый из которых приносит одинаковый доход $\lambda_{1}$ на одну купюру, вторая группа состоит из $G_{2}$ банков, каждый из которых приносит на одну купюру доход $\lambda_{2}$. Будем счптать, что параметры $\lambda_{1}$ и $\lambda_{2}$ удовлетворяют условию

$$
\lambda_{2}<\lambda_{1} \text {. }
$$

В рассматриваемой модели учтем, что кроме указанного дохода, пропорционального вкладу в банки, со вклада в первую группу банков взимается прогрессивный налог, который пропорционален квадрату вклада в данную группу, а кроме этого, на вклад во вторую группу банков начисляется дотация, пропорциональная квадрату вклада в эту группу банков. В таком случае доход вкладчика, в случае, когда его вклад в первую группу банков равен $N_{1}$, а вклад во вторую группу банков $-N_{2}=N-N_{1}$, выражается следуюшей формулой:

$$
\mathscr{E}\left(N_{1}, N_{2}\right)=\beta \lambda_{1} N_{1}+\beta \lambda_{2} N_{2}-\frac{\beta V_{1} N_{1}^{2}}{2 N}+\frac{\beta V_{2} N_{2}^{2}}{2 N},
$$

где $V_{1}>0, V_{2}>0$ - параметры, характеризующие размер налога и дотации соответственно. Будем далее считать, что выполняется следующие неравенства:

$$
V_{1}<\lambda_{1}-\lambda_{2}<V_{2} \text {. }
$$

Выполнение этих неравенств обеспечивает наличие у функции (47) двух максимумов на отрезке $N_{1} \in[0, N]$.

Все сказанное выше распространяется на случай, когда доход зависит от вкладов пропзвольным образом. Поэтому рассмотрим далее в модели с доходом вида (47) глобальный и локальные максимальные финансовые осреднения. Выражение (41) обобщается на этот случай следуюшим образом:

$$
\begin{aligned}
F\left(N_{1}\right)= & \beta \lambda_{1} N_{1}+\beta \lambda_{2}\left(N-N_{1}\right)-\frac{\beta V_{1} N_{1}^{2}}{2 N}+\frac{\beta V_{2}\left(N-N_{1}\right)^{2}}{2 N} \\
& +\ln \left(\frac{\left(G_{1}+N_{1}-1\right) !}{\left(G_{1}-1\right) ! N_{1} !}\right)+\ln \left(\frac{\left(G_{2}+N-N_{1}-1\right) !}{\left(G_{1}-1\right) !\left(N-N_{1}\right) !}\right) \\
& -\ln \left(\frac{\left(G_{1}+G_{2}+N-1\right) !}{\left(G_{1}+G_{2}-1\right) ! N !}\right)
\end{aligned}
$$

В этой формуле $N_{2}$ выражается через $N_{1}: N_{2}=N-N_{1}$. Соответственно глобальное финансовое осреднение дохода имеет вид:

$$
\mathbf{E}=\ln \left(\sum_{N_{1}=0}^{N} \exp \left(F\left(N_{1}\right)\right)\right)
$$

А локальное максимальное финансовое осреднение дохода, отвечающее вкладам $N_{1}^{0}$, $N_{2}^{0}=N-N_{1}^{0}$, выражается формулой

$$
\mathbf{E}\left(N_{1}^{0}\right)=\ln \left(\sum_{F\left(N_{1}\right) \geqslant F\left(N_{1}^{0}\right)} \exp \left(F\left(N_{1}\right)\right)\right) .
$$


Будем считать, что $G_{1}, G_{2}$ и $N_{1}^{0}$ зависят от $N$ так, что выполняются следуюшие равенства:

$$
\lim _{N \rightarrow \infty} \frac{G_{1}}{N}=g_{1}>0, \quad \lim _{N \rightarrow \infty} \frac{G_{2}}{N}=g_{2}>0, \quad \lim _{N \rightarrow \infty} \frac{N_{1}^{0}}{N}=w>0 .
$$

Исследуем в этом случае (50) и (51) при $N \rightarrow \infty$. Методы, развитые автором (операторные методы) позволяют найти асимптотику выражения (50) при $N \rightarrow \infty$, которая имеет вид:

$$
N\left(\max _{x \in[0,1]} f(x)+o(1)\right)
$$

где $f(x)$ - следуюшая функция:

$$
\begin{aligned}
f(x)= & \beta\left(\omega_{1}-\omega x+\frac{V x^{2}}{2}\right)+\left(g_{2}+x\right) \ln \left(1+\frac{x}{g_{2}}\right)-x \ln \left(\frac{x}{g_{2}}\right) \\
& +\left(g_{1}+1-x\right) \ln \left(1+\frac{1-x}{g_{1}}\right)-(1-x) \ln \left(\frac{1-x}{g_{1}}\right) \\
& -\left(1+g_{1}+g_{2}\right) \ln \left(1+g_{1}+g_{2}\right)+\left(g_{1}+g_{2}\right) \ln \left(g_{1}+g_{2}\right),
\end{aligned}
$$

здесь и далее использованы обозначения $\omega_{1}=\lambda_{1}-V_{1} / 2, \omega=\lambda_{1}-\lambda_{2}-V_{1}, V=$ $V_{2}-V_{1}$. Асимптотика выражения (51) при $N \rightarrow \infty$ находится аналогично. Если в точке $w$ функция (54) возрастает, т.е., например, выполняется неравенство $f^{\prime}(w)>0$, то при $N \rightarrow \infty$ главный член асимптотики локального максимального финансового осреднения (51) имеет вид:

$$
N \max _{x \in[w, 1]} f(x),
$$

если функция $f(x)$ является убывающей в точке $w$, т.е. при выполнении, например, неравенства $f^{\prime}(w)<0$, главный член асимптотики выражения (51) есть

$$
N \max _{x \in[0, w]} f(x),
$$

наконец, если в точке $w$ функция $f(x)$ имеет максимум - не важно глобальный или локальный - то главный член асимптотики выражения (51) есть $N f(w)$, а в случае, когда $w$-- точка минимума функции $f(x)$, асимптотика выражения (51) совпадает с асимптотикой (53) глобального финансового осреднения (50).

Исследуем теперь зависимость глобального максимума и экстремумов функции $f(x)$ на отрезке $x \in[0,1]$ от параметра $\beta$, считая, что параметры $V, \omega, g_{1}, g_{2}$ заданы. Из неравенств (48) следует, что $\omega>0$. Будем также считать далее, что

$$
\omega<V<2 \omega .
$$

Максимальное значение $f(x)$ достигается при

$$
x=x_{0}\left(\beta, V, \omega, g_{1}, g_{2}\right) \in(0,1), \quad \text { где } \quad x_{0}\left(\beta, V, \omega, g_{1}, g_{2}\right)
$$

является решением уравнения

$$
f^{\prime}\left(x_{0}\right)=\beta\left(-\omega+V x_{0}\right)-\ln \left(\frac{x_{0}}{g_{2}+x_{0}} \frac{g_{1}+1-x_{0}}{1-x_{0}}\right)=0 .
$$

Уравнение (58) всегда имеет по крайней мере одно решение, так как $f^{\prime}(x) \rightarrow+\infty$ при $x \rightarrow 0$ и $f^{\prime}(x) \rightarrow-\infty$ при $x \rightarrow 1$. Кроме того, в силу

$$
f^{(4)}(x)=2\left(\frac{1}{\left(g_{2}+x\right)^{3}}-\frac{1}{x^{3}}+\frac{1}{\left(g_{1}+1-x\right)^{3}}-\frac{1}{(1-x)^{3}}\right)<0,
$$

это уравнение не может иметь более трех корней. Легко показать, что при выполнении условий (57) уравнение (58) пмеет три решения, если $\beta$ достаточно велико, и одно 
решение, если $\beta$ достаточно мало. Асимптотика решений при $\beta \rightarrow \infty$ такова:

$$
\begin{aligned}
& x_{1} \approx \frac{g_{2}}{1+g_{1}} \exp (-\beta \omega), \\
& x_{2} \approx 1-\frac{g_{1}}{1+g_{2}} \exp (-\beta(V-\omega)), \\
& x_{3} \approx \frac{\omega}{V}+\frac{1}{V \beta} \ln \left(\frac{\omega / V}{g_{2}+\omega / V} \frac{g_{1}+1-\omega / V}{1-\omega / V}\right) .
\end{aligned}
$$

При $\beta \rightarrow 0$ асимптотика единственного решения уравнения (58) имеет вид

$$
x_{0}=\frac{g_{2}}{g_{1}+g_{2}}+\beta\left(\frac{V g_{2}}{g_{1}+g_{2}}-\omega\right) \frac{g_{1} g_{2}\left(1+g_{1}+g_{2}\right)}{\left(g_{1}+g_{2}\right)^{3}}+\mathrm{O}\left(\beta^{2}\right) .
$$

Таким образом видим, что в зависимости от параметров $\beta, V, \omega, g_{1}, g_{2}$ уравнение (58) может иметь три решения - в таком случае функция (54) имеет два максимума и один минимум - или меньшее число решений, тогда функция (54) имеет один максимум. Значения параметра $\beta$, при которых уравнение (58) имеет два решения, являются критическими, при приближении к этим значениям со стороны, где сушествуют три решения, два решения - один из максимумов и минимум функции (54) - приближаются друг к другу и превращаются в одно при достижении критических значений параметров, а при дальнейшем изменении параметров исчезают. Критические значения $\beta_{0}$ параметра $\beta$, при которых происходит описанная бифуркация решений уравнения (58), определяются из уравнения

$$
f^{\prime \prime}\left(x_{0}\right)=\beta_{0} V-\left(\frac{1}{x_{0}}-\frac{1}{g_{2}+x_{0}}+\frac{1}{1-x_{0}}-\frac{1}{g_{1}+1-x_{0}}\right)=0,
$$

где $x_{0}$ кроме того удовлетворяет уравнению (58). Заметим также, что при изменении параметра $\beta$ точка максимума функции (54) может измениться скачком. А именно, если кроме неравенств (57) выполнено также следуюшее неравенство:

$$
\frac{\omega}{V}<\frac{g_{2}}{g_{1}+g_{2}}
$$

то при некотором значении параметров глобальный и локальный максимумы меняются местами. Обозначим решения уравнения (58), отвечающие максимумам функции (54), $x_{1}\left(\beta, \omega, V, g_{1}, g_{2}\right)$ и $x_{2}\left(\beta, \omega, V_{2}, g_{1}, g_{2}\right)$, причем $x_{1}$ и $x_{2}$ непрерывно зависят от параметров $\beta, \omega, V, g_{1}, g_{2}$ и непрерывно дифференцируемы по этим параметрам. Критические значения $\beta_{c}$ параметра $\beta$ определяются из уравнения

$$
f\left(x_{1}\left(\beta_{c}, \omega, V_{2}, g_{1}, g_{2}\right)\right)=f\left(x_{2}\left(\beta_{c}, \omega, V_{2}, g_{1}, g_{2}\right)\right) .
$$

Если при приближении к критическому значению параметра $\beta$ абсолютный минимум функции (54) достигается при $x=x_{1}\left(\beta, V, \omega, g_{1}, g_{2}\right)$, то после пересечения параметрами критических значений абсолютный минимум функции (54) достигается при $x=x_{2}\left(\beta, V, \omega, g_{1}, g_{2}\right)$. При этом $\max f(x)$ является непрерывной функцией параметра $\beta$, а ее производная по $\beta$ в точке $\beta_{c}$ изменяется скачком.

Полученные результаты относительно максимума функции (54) приводят к практическим следствиям в рассматриваемой финансовой модели. Заметим, что условие (57) означает для рассматриваемой модели, что прогрессивный налог и доход достаточно зелики. А условие (63) означает, что во второй группе больше банков, чем в первой. Из формул (61) следует, что при очень больших значениях параметра $\beta$, т.е. при очень хорошем состоянии экономики, наиболее выгодно почти весь вклад держать в первой группе банков. Если выполнены неравенства (57) и (63), то при уменьшении параметра $\beta$ до критического значения $\beta_{c}$, определяемого из уравнения (64), ситуация меняется скачком и наиболее выгодным становится держать большую часть вклада во второй группе банков. С финансовой точки зрения это означает, что при ухудшении состояния экономики малая группа банков, приносящих большой доход, становится менее надежной, и максимальный доход можно получить при распределении вклада между двумя группами, причем вклад во вторую группу должен превосходить вклад 
в первую. Кроме того, описанная выше бифуркация решений уравнения (58) с точки зрения финансовой математики соответствует «пробою курса акций». Пусть при значенпи покупательной способности рубля $\beta$ функция $f(x)$ имеет три точки экстремума. В таком случае точка минимума, которую обозначим $x_{3}$, лежит между двумя точками максимума, которые обозначим $x_{1}$ и $x_{2}$. Пусть $x_{1}<x_{3}<x_{2}$. В этом случае из формул (55) и (56) следует, что главный член асимптотики при $N \rightarrow \infty$ локального усредненного финансового дохода (51) есть $N f\left(x_{1}\right)$ при $N_{1}<N x_{3}$ и $N f\left(x_{2}\right)$ при $N_{1}>N x_{3}$. То есть в данном случае все локальные усредненные финансовые доходы асимптотически принимают два значения. Если же при заданном параметре $\beta$ функция $f(x)$ пмеет только один экстремум - глобальный максимум - то главный член асимптотікиі любого локального усредненного финансового дохода (51) равен $N \max f(x)$. То есть аспмптотически все локальные усредненные доходы в данном случае принимают одно значение. Таким образом, при $\beta=\beta_{0}$ происходит исчезновение (появление) одного из асимптотических значений локальных усредненных финансовых доходов. Это явление и есть пробой курса акций.

В заключение автор хотел бы выразить глубокую благодарность В. Н. Батурину II С. Г. Лебедеву за весьма плодотворную дискуссию по поводу нелинейного характера осредненія, а также Г. В. Ковалю за большую помошь в подготовке этой работы.

\section{СПИСОК ЛИТЕРАТУРЫ}

1. Мушик Э., Мюллер П. Методы принятия технических решений. М.: Мир, 1990, $208 \mathrm{c}$.

2. Горелик В. А., Ушаков И. А. Исследование операций. М.: Машиностроение, 1986, 286 c.

3. Современное состояние теории исследования операций. Под ред. Н. Н. Моисеева. М.: Наука, 1986, 464 с.

4. Моисеев Н.Н. Математические задачи системного анализа. М.: Наука, 1981, $488 \mathrm{c}$.

5. Фон Нейман Дж., Моргенштерн О. Теория игр и экономическое поведение. М.: Наука, 1970, 708 c.

6. Интрилигатор М. Математические методы оптимизации и экономическая теория. М.: Прогресс, 1975,600 с.

7. Фишберн П. Теория полезности для принятия решений. М.: Наука, 1978, 352 с.

8. Гермейер Ю. Б. Введение в теорию исследования операций. М.: Наука, 1971, 384 с.

9. Маслов В. П. Экспертизы и эксперименты. - Новый мир, 1991, № 1, с. 243-252.

10. Мизес Р. Вероятность и статистика. М.-Л.: Госиздат, 1930.

11. Church A. On the concept of random sequence. - Bull. Amer. Math. Soc., 1940, v. 46, № 2, p. 130-135.

12. Ville J. Etude critique de la notion de collectif. Paris: Gauthier-Villars, 1939.

13. Kolmogorov A. N. On tables of random numbers. - Sankhyā. The Indian Journal of Statistics, Ser. A, 1963, v. 25, № 4, p. 369-376. (Русский перевод: О таблицах случайных чисел. - Семиотика и информатика, М.: ВИНИТИ, 1982, вып. 2 (второй выпуск за 1981 г.), с. 3-13.)

14. Колмогоров А.Н. Три подхода к определению понятия «количества информации». - Проблемы передачи информации, 1965, т. 1, № 1, с. 3-11.

15. Колмогоров А.Н. К логическим основам теории информации и теории вероятностей. - Проблемы передачи информации, 1969, т. 5, № 3, с. 3-7.

16. Колмогоров $A . H$. Комбинаторные основания теории информации и исчисления вероятностей. - Успехи матем. наук, 1983, т. 38, № 4, с. 27-36.

17. Маслов В.П. Среднестатистическая информация относительно выпуклой функции и общие линейные уравнения для пространства min, max. - Докл. PAH, 2003, т. 393, № 1, c. 1-5. 